\title{
The Emerging Roles of Tripartite Motif Proteins (TRIMs) in Acute Lung Injury
}

\author{
Yingjie Huang $\mathbb{D}^{1}$, Yue Xiao $\mathbb{D}^{2},{ }^{2}$ Xuekang Zhang $\mathbb{D}^{1},{ }^{1}$ Xuan Huang $\mathbb{D}^{3}$, and Yong Li $\mathbb{D}^{1}$ \\ ${ }^{1}$ Department of Anesthesiology, The First Affiliated Hospital of Nanchang University, Nanchang, China \\ ${ }^{2}$ The First Clinical Medical College, Nanchang University, Nanchang 330006, China \\ ${ }^{3}$ The National Engineering Research Center for Bioengineering Drugs and the Technologies, Institute of Translational Medicine, \\ Nanchang University, Nanchang, China
}

Correspondence should be addressed to Xuekang Zhang; kang7139@163.com, Xuan Huang; huangxuan@ncu.edu.cn, and Yong Li; liyong@ncu.edu.cn

Received 12 July 2021; Accepted 9 October 2021; Published 19 October 2021

Academic Editor: Elizabeth Soares Fernandes

Copyright (C) 2021 Yingjie Huang et al. This is an open access article distributed under the Creative Commons Attribution License, which permits unrestricted use, distribution, and reproduction in any medium, provided the original work is properly cited.

\begin{abstract}
Acute lung injury (ALI) is an inflammatory disorder of the lung that causes high mortality and lacks any pharmacological intervention. Ubiquitination plays a critical role in the pathogenesis of ALI as it regulates the alveolocapillary barrier and the inflammatory response. Tripartite motif (TRIM) proteins are one of the subfamilies of the RING-type E3 ubiquitin ligases, which contains more than 80 distinct members in humans involved in a broad range of biological processes including antivirus innate immunity, development, and tumorigenesis. Recently, some studies have shown that several members of TRIM family proteins play important regulatory roles in inflammation and ALI. Herein, we integrate emerging evidence regarding the roles of TRIMs in ALI. Articles were selected from the searches of PubMed database that had the terms "acute lung injury," "ubiquitin ligases," "tripartite motif protein," "inflammation," and "ubiquitination" using both MeSH terms and keywords. Better understanding of these mechanisms may ultimately lead to novel therapeutic approaches by targeting TRIMs for ALI treatment.
\end{abstract}

\section{Introduction}

Acute lung injury (ALI) is an acute hypoxic respiratory insufficiency caused by various direct (pulmonary) or indirect (extrapulmonary) injuries including sepsis syndrome, ischemia-reperfusion, pneumonia, and mechanical ventilation, which leads to the destruction of the barrier of alveolar epithelial cells and capillary endothelial cells, resulting in overinfiltration of inflammatory cells and diffuse pulmonary interstitial and alveolar edema [1]. In 1994, the diagnostic criteria of ALI were put forward by the American-European Consensus Conference: an acute onset; oxygenation index $\left(\mathrm{PaO}_{2} / \mathrm{FiO}_{2}\right)>$ $200 \mathrm{~mm} \mathrm{Hg}$ and $<300 \mathrm{~mm} \mathrm{Hg}(1 \mathrm{mmHg}=0.133 \mathrm{kPa})$; patchy shadows in both lungs on the chest X-ray; pulmonary artery wedge pressure $\leq 18 \mathrm{mmHg}$ or no clinical evidence of left atrial hypertension; etc. [2]. Due to the lack of drug intervention, ALI remains a significant cause of morbidity and mortality in the critically ill patient population [2]. More severe situations with
$\mathrm{PaO}_{2} / \mathrm{FiO}_{2} \leq 200 \mathrm{~mm} \mathrm{Hg}$, ALI turns to the worse stage acute respiratory distress syndrome (ARDS) [3].

Pathological hallmarks of ALI are injury to the vascular endothelium/alveolar epithelium, activation of innate immune response, and enhanced coagulation [4]. Exposure to several risk factors (i.e., pneumonia, sepsis, and shock) firstly leads to endothelial and/or epithelial monolayers damage, increasing permeability and impairing their barrier function [4]. A large amount of protein-rich fluid and inflammatory cells leaks into the alveoli and lung interstitium, resulting in pulmonary edema, neutrophil infiltration, cytokine and reactive oxygen species-mediated inflammation coagulation disorders, and pulmonary fibrosis [5]. Notedly, mast cells (MCs) and polymorphonuclear neutrophils (PMNs) are the main inflammatory cells, which play a critical role in the pathogenesis of ALI $[6,7]$. MC activation induces tryptase release to trigger ALI [8], which is supported by the finding that MCs "stabilizers" can reduce ALI severity [9]. 
At present, there is still no effective pharmaceutical intervention for ALI; mechanical ventilation is the main approach to prevent respiratory failure and, combined with intensive care support, could improve health condition [10]. However, mechanical ventilation can exacerbate preexisting lung injury or even induce de novo injury in healthy lungs, which is called ventilator-induced lung injury (VILI) $[11,12]$. In recent years, researchers have paid close attention to the identification of new routes at cellular level which could provide a better understanding of the physiopathology of ALI, but the precise cellular and molecular underlying mechanisms are still to be fully elucidated. Chen et al. recently summarized and introduced the role of lncRNAs in ALI in detail [13]. However, emerging evidence points out to the ubiquitination which functions as an important regulator in the pathobiology of ALI since it regulates the proteins evolved in the modulation of the alveolocapillary barrier and inflammatory response, opening a highly promising research field for the treatment of lung diseases [14].

\section{Ubiquitination in ALI}

Ubiquitination is the major protein posttranslational modification in cells by which ubiquitin (Ub) covalently attached the target protein for degradation through the 26S proteasome or lysosome or nonproteolytic modifications [15]. It plays crucial roles in diverse biological processes such as DNA repair, cell proliferation, signal transduction, apoptosis, and inflammation, whose dysregulation leads to many diseases [16]. However, bacterial infection or inflammatory stimulation often disrupts the process of protein ubiquitination. We and some other investigators have shown that expressions of some E3 Ub ligases were altered by infection or inflammation thus affecting the levels and functions of their target proteins. Thus, uncovering new E3 Ub ligaserelated molecular mechanisms and signaling pathways will provide a unique opportunity for the potential design of new strategies to alleviate ALI.

2.1. The Ubiquitin System. As a multicomponent regulatory system, the Ub system is composed of three types of Ub enzymes, which is a highly controlled mechanism of protein degradation and turnover in cells, starting with approximately $8 \mathrm{kDa}$ monomeric Ub [17]. Ub is activated by a Ub-activating enzyme (E1) in an adenosine-triphosphate- (ATP-) dependent manner and then conjugated by a ubiquitin conjugating enzyme (E2), finally resulting in transfer of $\mathrm{Ub}$ to an internal lysine of the substrate protein by an E3 ligase [18]. To date, there are only two E1 enzymes (UBA1 and UBA6) [19], around 40 E2 enzymes [20], but more than 600 E3 ligases [21] existed in the human genome. Although the addition of $\mathrm{Ub}$ moieties to specific residues on a substrate protein is partly because of E2/E3 enzymes pairings, E3 ligases were considered the predominant source of substrate specificity [22].

2.2. E3 Ligases. As a direct mediator of substrate tagging and ubiquitin chain elongation, E3 ligase is considered an essential component in the ubiquitin system that determines substrate specificity. In human genome, more than 600 putative
E3 ligases have been identified [21]. There are three major kinds of E3 ligases divided by the molecular structure and functional mechanism, including HECT (homologous to the E6-associated protein carboxyl-terminus) domain family, RING (really interesting new gene) finger family, and the RBR (RING in-between-RING) E3 ubiquitin ligases [23]. HECT E3 ligase contains an N-terminal lobe which is responsible for $\mathrm{E} 2$ binding and substrate recognition, and a C-terminal HECT domain containing a catalytic cysteine that receives and passes an ubiquitin molecule from the E2 enzyme before conjugating ubiquitin to a substrate protein [24]. RING finger E3 ligases constitute the largest family of E3 ligases which are characterized based on the presence of a RING domain [25]. Interestingly, the canonical RING domain is a type of zinc finger with a RING fold structure while another type is the U-box domain which possesses the same RING fold but without zinc [26]. Unlike HECT E3 ligase, RING finger E3 ligase mediates the direct transfer of ubiquitin to a substrate protein by binding to a ubiquitincharging E2 enzyme as a scaffold [27]. Notably, RING E3 ligases function either as monomers (e.g., c-CBL, E4B), homodimers (e.g., cIAP, CHIP), heterodimers (e.g., Mdm2$\mathrm{MdmX}$ ), or large multisubunit complexes, such as the Cullin-RING ligases (CRLs), which make up a distinct subtype characterized by their common Cullin scaffold protein [23]. RBR E3 ligases contain two RING domains (RING1 and RING2) with an in-between-RING (IBR) domain and share the common features of both HECT and RING finger E3 ligases which function as a hybrid of these two types of E3 ligases [28]. Specifically, the RING1 domain binds to ubiquitin-loaded E2 and transfers ubiquitin onto the RING2 domain at a catalytic cysteine residue before conjugation to the substrate protein [29].

2.3. Role of E3 Ligases in ALI. Although ubiquitination has been reported to play a pivotal role in multiple biological functions, its function in ALI remains poorly understood. Recently, the key regulative role of ubiquitination in ALI has been mentioned increasingly [14]. Of them, most studies have been focused on the E3 ubiquitin ligases and the conventional K48-ubiquitination which leads to the substrate proteins degradation via the $26 \mathrm{~S}$ proteasome [30]. Accumulating evidence has demonstrated that E3 ligase plays a critical role in the pathobiology of ALI since it modulates critical proteins involved in the alveolocapillary barrier and the inflammatory response [14].

Tight junctions form a highly selective diffusion barrier between endothelial cells and epithelial cells by preventing most dissolved molecules and ions from passing freely through the paracellular pathway [31]. The function impairment of tight junction is a sign of ALI [32]. E3 ligase Itch, a member of the HECT Ub ligases, could directly interact with and degrade the tight junction-specific protein occludin [33] via ubiquitination. E-cadherin, a well-studied member of the classical cadherin family, is a central component in the cellcell adhesion junction and plays a critical role in maintaining cell polarity and the integrity of epithelial cells [34]. Dysfunction of E-cadherin contributes to the pathogenesis of ALI [35]. A RING finger E3 ligase Hakai induces 
E2-dependent ubiquitination and endocytosis of E-cadherin complex in epithelial cells [36]. Recently, Dong et al. found that the HECT E3 ligase Smurf2 induced $\mu$-opioid receptor 1 (MOR1) degradation in the ubiquitin-proteasome system in lung epithelial cells, and MOR1 has a potential effect in lung repair and remodeling after ALI [37].

E3 ligase Cblb inhibits the MyD88-dependent Toll-like receptor 4 (TLR4) signaling and attenuates acute lung inflammation induced by polymicrobial sepsis [38]. The ST2L receptor for interleukin 33 (IL-33) mediates pulmonary inflammation during ALI, which is bound and ubiquitinated by FBXL19, a member of the Skp1-Cullin-F-box family of E3 ubiquitin ligases [39]. In addition, E3 ligase FBXO3 targets the TRAF inhibitor FBXL2 for its destabilization and potently stimulates cytokine release, leading to changes in lung permeability, alveolar edema, and ALI [40]. FBXO17 has been described as an E3 ligase that recognizes and mediates the ubiquitination and degradation of GSK $3 \beta$ to reduce inflammatory responses in lung epithelial cells after LPS injury [41]. Most recently, Lear et al. reported that E3 ligase KIAA0317 targets SOCS2 for ubiquitination and degradation by the proteasome and exacerbates pulmonary inflammation [42]. These studies have proved that ubiquitin-proteasome system especially E3 ligases is closely related to the pathogenesis of lung injury.

\section{TRIMs in ALI}

TRIM proteins are regarded as a subfamily of the RING finger E3 ligase, which contain more than 80 distinct members in humans [43]. TRIMs are composed by conserved three zinc-binding domains, an N-terminal RING domain, one or two B-boxes, and a central coiled-coiled domain (CDD) [44]. We recently found that TRIM65 selectively targeted vascular cell adhesion molecule 1 (VCAM-1) and promoted its ubiquitination and degradation, by which it critically controlled the duration and magnitude of pulmonary inflammation in ALI [45]. Particularly, Whitson and his colleagues reported that TRIM72 (also known as MG53) could function as a novel therapeutic protein to treat ALI [46]. Here, we discuss our current understanding of TRIMs as E3 ligases that executes its effector functions in ALI (Table 1).

3.1. TRIM8. TRIM8, as a member of TRIM family, has a common structural feature of a typical RBCC motif as well as a monopartite nuclear localization signal (NLS), which allows shuttling and functioning into the nucleus [47]. It has been reported that TRIM8 can regulate NF- $\kappa$ B signaling both in the nucleus and cytoplasm: TRIM8 inhibited PIAS3mediated negative regulation of p65 to enhance NF- $\kappa \mathrm{B}$ activity in the nucleus [48] and can also positively regulate NF- $\kappa$ B pathway through k63-linked polyubiquitination of cytoplasmic protein TAK1 [49]. TRIM8 is ubiquitously expressed in human and mouse tissues, which has higher expression levels in the central nervous system, kidney, and lens, and lower expression level in digestive tract [44]. TRIM8 plays a key role in the immune response and participates in various fundamental biological processes such as cell survival, apoptosis, autophagy, differentiation, inflammation, and carcinogenesis [50].

Recently, studies have revealed that TRIM8 is involved in the regulation of sepsis and ALI. TRIM8 was significantly upregulated in LPS (lipopolysaccharide) sepsis-induced acute hepatic injury (AHI), which was a direct target of miR-373-3p [51]. Moreover, inhibition of TRIM8 by downregulation of long noncoding RNA (lncRNA) LINC00472, which served as a sponge for miR-373-3p and negatively regulated its expression, could reduce sepsis-induced expression of main proinflammatory cytokines such as IL-6, IL-10, and TNF- $\alpha$ [51]. Xiaoli et al. found that TRIM8 was increased in a time-dependent manner during LPS-induced ALI, promoting inflammatory response and ROS generation via the inactivation of $\mathrm{p}$-AMPK $\alpha$. In addition, suppression of TRIM8 markedly downregulated mRNA levels of interleukin- $1 \beta$ (IL-1 $\beta$ ), IL-6, and tumor necrosis factor- $\alpha$ (TNF- $\alpha$ ) in lung epithelial cells mainly through blocking the NF- $\kappa \mathrm{B}$ signaling pathway and alleviated oxidative stress by regulating Nrf2 signaling and heme oxygenase-1 (HO-1) expression [52]. Although TRIM8 has been revealed to play an important role in acute lung injury, precise regulatory mechanisms such as whether it depends on the activity of E3 ubiquitin ligase and its specific target proteins need to be further clarified.

3.2. TRIM14. TRIM14 was originally known as KIAA0129 [53], and its overexpression was first found in human immunodeficiency virus- (HIV-) infected human and simian non-Hodgkin's lymphoma infected with simian immunodeficiency virus (SIV) [54, 55]. TRIM14 is a noncanonical member of the TRIM family, since it lacks the N-terminal RING domain of the typical RBCC motif which can exert an E3 ubiquitin ligase [56]. Studies have shown that TRIM14 can perform various functions via partners with which directly interact with its PRYSPRY domain [57]. Interestingly, TRIM14 was reported to be an important mediator of antiviral immunity both in DNA virus and double-stranded RNA virus infection $[58,59]$. Furthermore, several groups found that TRIM14 may be involved in tumorigenesis [60-64].

Recently, we found that TRIM14 was overexpressed in human vascular endothelial cells (ECs) and markedly induced by inflammatory stimuli such as LPS [65]. TRIM14 was a new positive regulator of endothelial activation via activating the NF- $\kappa \mathrm{B}$ signaling pathway, which can directly bind to the promoter of TRIM14 gene and control its transcription [65]. Zhou et al. revealed that TRIM14 underwent Lys-63-linked autopolyubiquitination at Lys-365 and served as a platform and recruited NEMO to the mitochondrial antiviral signal (MAVS) complex, leading to the activation of interferon regulatory factor 3 (IRF3) and NF- $\kappa$ B signaling in human lung epithelial cells, which boost antiviral innate immune response [66]. TRIM14 also can recruit USP14 to cleave the K63-linked ubiquitin chain at lysine 332/338/341 of $\mathrm{p} 100 / \mathrm{p} 52$, hinder the recognition of receptor $\mathrm{p} 62$, and inhibit the autophagy degradation of $\mathrm{p} 100 / \mathrm{p} 52$, thus promoting the atypical activation of NF- $\kappa \mathrm{B}$ in vivo and in vitro [67]. Considering endothelial inflammation and dysfunction play a prominent role in development of ALI and $\mathrm{NF}-\kappa \mathrm{B}$ is a central transcriptional factor in ALI, it is 
TABLE 1: Role of TRIMs in acute lung inflammation.

\begin{tabular}{lcccc}
\hline TRIMs & Models & Cell types & Mechanisms & Ref No. \\
\hline \multirow{2}{*}{ TRIM8 } & LPS-induced AHI & Human liver cells & LINC00472/miR-373-3p/TRIM8 axis & {$[51]$} \\
& LPS-induced ALI & Lung epithelial cells & p-AMPK $\alpha /$ NF- $\kappa$ B/Nrf2/ROS/HO-1 axis & {$[52]$} \\
TRIM14 & LPS-induced ALI & Human vascular endothelial cells & NEMO/TAK1/NF- $\kappa$ b/TRIM14 pathway & {$[63]$} \\
TRIM21 & LPS-induced ALI & Lung microvascular endothelial cells & NF- $\kappa$ B signaling & {$[79]$} \\
TRIM65 & ALI & Human vascular endothelial cells & VACM-1 ubiquitination and degradation & {$[45]$} \\
& Ischaemia-reperfusion and & Lung epithelial cells & Cell membrane repair & {$[97]$} \\
& overventilation-induced ALI & Macrophage & NF- $\kappa$ B signaling & {$[98,106]$} \\
TRIM72 & Influenza virus -induced ALI & Lung tissue & Inhibition of pyroptosis & {$[46]$} \\
& Hemorrhagic shock/contusive ALI & Human bronchial epithelial cells & Cell membrane repair & \\
\hline
\end{tabular}

suggested that TRIM14 may be involved in the pathological process of ALI, which needs further study.

3.3. TRIM21. TRIM21, also known as Ro52, has a typical RBCC motif and E3 ligase activity [68]. It is broadly expressed in most human tissues and cells and predominantly expressed in hematopoietic cells and endothelial and epithelial cells [69]. TRIM21 was identified as a major autoantigen in autoimmune diseases including Sjögren's syndrome, systemic lupus erythematosus (SLE), and rheumatoid arthritis [70-72]. Later studies revealed that TRIM21 is a highly conserved IgG receptor with high cytoplasmic affinity and specificity $[73,74]$, which can be induced by interferon to exert antiviral effect [75]. TRIM21 serves as a multifaceted regulator in viral immunity and can not only promote the production of type I interferon [76] and triggers an innate immune response via RIG-1 and cGAS sensing [77] but also negatively regulate innate immunity by targeting and degrading the viral DNA sensor DEAD (Asp-GluAla-Asp) box polypeptide 41 (DDX41) [78]. The biological function and application of TRIM21 in antiviral immunity are described in detail in other reviews [79].

Using TRIM21-deficient mice, Yoshimi and colleagues found that TRIM21 is a negative regulator of NF- $\kappa$ B-dependent proinflammatory cytokine production induction in fibroblasts after TLR ligands (poly(I:C), CpG, and LPS) stimulation [80]. In addition, TRIM21 deletion can lead enhanced production of proinflammatory cytokines and systemic autoimmunity though the IL-23-Th17 pathway [81]. Recently, Li et al. reported that TRIM21 exhibited an antiinflammatory property against LPS-induced lung endothelial dysfunction and monocytes adhesion to endothelial cells [82]. TRIM21 can be monoubiquitylated and lysosomal degraded in response to LPS and may contribute to the pathogenesis of ALI [52]. TRIM21 can be used as a therapeutic target for endothelial dysfunction induced by sepsis, such as acute lung injury [83]. However, whether ubiquitination of TRIM21 is dependent on its phosphorylation and the specific phosphorylation or ubiquitination sites needs to be further clarified.

3.4. TRIM65. Human TRIM65 is a 517-amino acid protein, containing a N-terminal RING domain, a B-box, a coiledcoil domain, and a SPRY domain, is first known as a gene associated with white matter lesions [84, 85]. Using a systematic discovery-type proteomic analysis, $\mathrm{Li}$ et al. found that TRIM65 can negatively regulate miRNA-mediated mRNA translation inhibition through ubiquitination and subsequent degradation of trinucleotide repeat containing six (TNRC6) [86, 87]. Like other TRIMs, TRIM65 also participates in the antiviral innate immune response by ubiquitination of MDA5 [88, 89]. Over the years, several reports suggest that TRIM65 acts as a ubiquitin E3 ligase, targeting p53, ANXA2, Axin1, and ARHGAP35 to regulate carcinogenesis [90-93]. Most recently, Liu et al. published a review to introduce TRIM65 in white matter lesions, innate immunity and tumor [94].

We recently found that TRIM65 may control the magnitude and duration of LPS-induced lung inflammation and injury [45]. TRIM65-deficient (TRIM65 ${ }^{-/}$) mice are more sensitive to LPS-induced death due to sustained and severe pulmonary inflammation. Further studies showed that monocytes/macrophages were higher in the BAL from

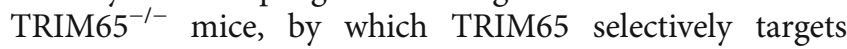
vascular cell adhesion molecule 1 (VCAM-1) and directly induces its ubiquitination degradation in endothelial cells. It is worth noting that TRIM65 does not affect the MAPK and NF- $\kappa \mathrm{B}$ signaling pathways in ALI, although some studies have revealed that TRIM65 can activate the Erk1/2 pathway $[95,96]$, which suggests that TRIM65 has diverse functions in different cells and under distinct pathological conditions. Furthermore, TRIM65 is enriched in endothelial cells and declined at the early stage during endothelial activation; the mechanisms that precisely regulate TRIM65 levels in endothelial inflammation remain unknown. Further studies are necessary to understand the regulatory mechanisms that control TRIM65 expression.

3.5. TRIM72. TRIM72 (also known as MG53) is composed of a typical TRIM family protein RBCC structure and a PRY-SPRY subdomain which is mainly expressed in cardiac and skeletal muscle, as well as in renal and alveolar epithelial cells, monocyte, and macrophages with detectable amount level [97-100]. Cai et al. first revealed that TRIM72 acted as a key component of the sarcolemma cell plasma membrane repair machinery [101]. Upon the membrane injurious stimuli, TRIM72 oligomerized by oxidizing the thiol group of cysteine at position 242 and a leucine zipper 
motif to induce the intracellular vesicles coated with TRIM72 to nucleate at the injured site, resulting in resealing the damaged membrane [102, 103]. At the membrane, TRIM72 protein binds to phosphatidyl serine to mediate the recruitment of vesicles at the injured site [104]. Interestingly, TRIM72 can be secreted and circulate throughout the entire body to reach all tissues and organs, allowing the recombinant TRIM72 protein to have therapeutic benefit in treatment of injuries to multiple tissues, such as the heart, kidney, lung, brain, liver, skin, skeletal muscle, and cornea [105].

Ablation of the TRIM72 gene leads to increased susceptibility to ischemia-reperfusion and overventilation-induced ALI in mice [97]. Recently, Sermersheim and his colleagues found that knockdown of TRIM72 in macrophages results in activation of NF- $\kappa \mathrm{B}$ signaling and increased inflammatory factor interleukin- $1 \beta$ upon influenza virus infection, and knockout of TRIM72 promotes $\mathrm{CD}^{+} 5^{+}$cells infiltration and IFN $\beta$ elevation in the lung [98]. Kenney et al. found that exogenous injection of recombinant human TRIM72 protein could protect ALI caused by lethal influenza virus infection [106]. Recombinant TRIM72 protein significantly decreased the level of inflammatory cytokines of IFN $\beta$, IL-6, and IL-1 $\beta$ and infiltrating $\mathrm{CD} 11 \mathrm{~b}^{+}$lymphocytes in lung tissues [106]. It is reported that intravenous (IV) delivery or inhalation of recombinant human TRIM72 protein reduces symptoms in rodent models of ALI and emphysema [97]. The extracellular recombinant protein protects cultured lung epithelial cells against anoxia/reoxygenation-induced injuries [97]. Most recently, Whitson et al. had evaluated the therapeutic benefits of recombinant human TRIM72 protein in porcine models of ALI and found that recombinant TRIM72 protein can mitigate lung injury in the porcine model of combined hemorrhagic shock/contusive lung injury and reduce warm ischemia-induced injury to the isolated porcine lung through ex vivo lung perfusion administration [46]. These findings revealed that TRIM72 plays a critical role in ALI, and exogenous-recombinant TRIM72 protein may be a shelf stable therapeutic agent with the potential to restore lung function and lessen the impact of ALI.

\section{Conclusions}

TRIMs are a wide and well-conserved family of proteins defined as a subfamily of the RING-type E3 Ub ligases, which have been implicated in a broad range of biological processes including antiviral immunity, cell differentiation, development, and carcinogenesis. Accumulating evidence has shown that several TRIM members have unique and vital roles in ALI using distinct mechanisms (Table 1). Particularly, the regulation of ALI by targeting cell membrane repair has been a focus of intense research in the last few years. Interestingly, systemically administered recombinant human TRIM72 proteins could recognize injury to both epithelial and endothelial layers in the lung, which can effectively preserve lung structure and function in ALI. TRIM72 will be one of the most promising therapeutic agents with the potential to restore lung function and lessen the impact of ALI. Further work is needed to understand full contribution of TRIMs including discovered and undiscovered mem- bers to ALI. Identification of TRIM proteins with the potential to serve as therapeutic targets of ALI may help to novel drug development of ALI treatment.

\section{Data Availability}

No data were used to support this study.

\section{Conflicts of Interest}

The authors declare that they have no conflicts of interest.

\section{Acknowledgments}

This work was supported by the grants from the National Natural Science Foundation of China (31760329, 31960147, 32170793, and 82160133) and Research and Training Fund for Young Talents in the First Affiliated Hospital of Nanchang University (YFYPY202003).

\section{References}

[1] G. D. Rubenfeld, E. Caldwell, E. Peabody et al., "Incidence and outcomes of acute lung injury," The New England Journal of Medicine, vol. 353, no. 16, pp. 1685-1693, 2005.

[2] G. R. Bernard, A. Artigas, K. L. Brigham et al., "The American-European Consensus Conference on ARDS. Definitions, mechanisms, relevant outcomes, and clinical trial coordination," American Journal of Respiratory and Critical Care Medicine, vol. 149, no. 3, pp. 818-824, 1994.

[3] L. B. Ware and M. A. Matthay, "The acute respiratory distress syndrome," The New England Journal of Medicine, vol. 342, no. 18, pp. 1334-1349, 2000.

[4] V. Fanelli and V. M. Ranieri, "Mechanisms and clinical consequences of acute lung injury," Annals of the American Thoracic Society, vol. 12, Supplement 1, pp. S3-S8, 2015.

[5] E. R. Johnson and M. A. Matthay, "Acute lung injury: epidemiology, pathogenesis, and treatment," Journal of Aerosol Medicine and Pulmonary Drug Delivery, vol. 23, no. 4, pp. 243-252, 2010.

[6] H. Virk, G. Arthur, and P. Bradding, "Mast cells and their activation in lung disease," Translational Research, vol. 174, pp. 60-76, 2016.

[7] T. R. Martin, "Neutrophils and lung injury: getting it right," The Journal of Clinical Investigation, vol. 110, no. 11, pp. 1603-1605, 2002.

[8] X. Gan, D. Liu, P. Huang, W. Gao, X. Chen, and Z. Hei, "Mast-cell-releasing tryptase triggers acute lung injury induced by small intestinal ischemia-reperfusion by activating PAR-2 in rats," Inflammation, vol. 35, no. 3, pp. 1144$1153,2012$.

[9] C. Chen, Z. Zhang, F. Tan et al., "Stabilizing mast cells improves acute lung injury after orthotopic liver transplantation via promotion of apoptosis in polymorphonuclear neutrophils," American Journal of Physiology. Lung Cellular and Molecular Physiology, vol. 320, no. 2, pp. L266-L275, 2021.

[10] M. A. Matthay, D. McAuley, and L. B. Ware, "Clinical trials in acute respiratory distress syndrome: challenges and opportunities," The Lancet, vol. 5, no. 6, pp. 524-534, 2017.

[11] D. Dreyfuss and G. Saumon, "Ventilator-induced lung injury: lessons from experimental studies," American Journal of 
Respiratory and Critical Care Medicine, vol. 157, no. 1, pp. 294-323, 1998.

[12] E. K. Wolthuis, A. P. J. Vlaar, G. Choi, J. J. T. H. Roelofs, N. P. Juffermans, and M. J. Schultz, "Mechanical ventilation using non-injurious ventilation settings causes lung injury in the absence of pre-existing lung injury in healthy mice," Critical Care, vol. 13, no. 1, p. R1, 2009.

[13] C. Chen, Y. He, Y. Feng, W. Hong, G. Luo, and Z. Ye, "Long non-coding RNA review and implications in acute lung inflammation," Life Sciences, vol. 269, p. 119044, 2021.

[14] N. D. Magnani, L. A. Dada, and J. I. Sznajder, "Ubiquitinproteasome signaling in lung injury," Translational Research, vol. 198, pp. 29-39, 2018.

[15] K. N. Swatek and D. Komander, "Ubiquitin modifications," Cell Research, vol. 26, no. 4, pp. 399-422, 2016.

[16] R. B. Damgaard, "The ubiquitin system: from cell signalling to disease biology and new therapeutic opportunities," Cell Death and Differentiation, vol. 28, no. 2, pp. 423-426, 2021.

[17] J. Callis, "The ubiquitination machinery of the ubiquitin system," The Arabidopsis Book, vol. 12, article e0174, 2014.

[18] C. C. Goetzke, F. Ebstein, and T. Kallinich, "Role of proteasomes in inflammation," Journal of Clinical Medicine, vol. 10, no. 8, p. 1783, 2021.

[19] J. Jin, X. Li, S. P. Gygi, and J. W. Harper, "Dual E1 activation systems for ubiquitin differentially regulate E2 enzyme charging," Nature, vol. 447, no. 7148, pp. 1135-1138, 2007.

[20] M. D. Stewart, T. Ritterhoff, R. E. Klevit, and P. S. Brzovic, "E2 enzymes: more than just middle men," Cell Research, vol. 26, no. 4, pp. 423-440, 2016.

[21] W. Li, M. H. Bengtson, A. Ulbrich et al., "Genome-wide and functional annotation of human E3 ubiquitin ligases identifies MULAN, a mitochondrial E3 that regulates the organelle's dynamics and signaling," PLoS One, vol. 3, no. 1, article e1487, 2008.

[22] A. Hershko and A. Ciechanover, "The ubiquitin system," Annual Review of Biochemistry, vol. 67, no. 1, pp. 425-479, 1998.

[23] N. Zheng and N. Shabek, "Ubiquitin ligases: structure, function, and regulation," Annual Review of Biochemistry, vol. 86, no. 1, pp. 129-157, 2017.

[24] J. M. Huibregtse, M. Scheffner, S. Beaudenon, and P. M. Howley, "A family of proteins structurally and functionally related to the E6-AP ubiquitin-protein ligase," Proceedings of the National Academy of Sciences of the United States of America, vol. 92, no. 11, p. 5249, 1995.

[25] K. L. Lorick, J. P. Jensen, S. Fang, A. M. Ong, S. Hatakeyama, and A. M. Weissman, "RING fingers mediate ubiquitinconjugating enzyme (E2)-dependent ubiquitination," Proceedings of the National Academy of Sciences of the United States of America, vol. 96, no. 20, pp. 11364-11369, 1999.

[26] C. W. Vander Kooi, M. D. Ohi, J. A. Rosenberg et al., “The Prp19 U-box crystal structure suggests a common dimeric architecture for a class of oligomeric E3 ubiquitin ligases," Biochemistry, vol. 45, no. 1, pp. 121-130, 2006.

[27] F. E. Morreale and H. Walden, "Types of ubiquitin ligases," Cell, vol. 165, no. 1, pp. 248-248.e1, 2016.

[28] C. E. Berndsen and C. Wolberger, "New insights into ubiquitin E3 ligase mechanism," Nature Structural \& Molecular Biology, vol. 21, no. 4, pp. 301-307, 2014.

[29] D. M. Wenzel, A. Lissounov, P. S. Brzovic, and R. E. Klevit, "UBCH7 reactivity profile reveals parkin and HHARI to be
RING/HECT hybrids," Nature, vol. 474, no. 7349, pp. 105108, 2011.

[30] I. Vadász, C. H. Weiss, and J. I. Sznajder, "Ubiquitination and proteolysis in acute lung injury," Chest, vol. 141, no. 3, pp. 763-771, 2012.

[31] C. Zihni, C. Mills, K. Matter, and M. S. Balda, "Tight junctions: from simple barriers to multifunctional molecular gates," Nature Reviews Molecular Cell Biology, vol. 17, no. 9, pp. 564-580, 2016.

[32] Y. Liu, S. Mu, X. Li, Y. Liang, L. Wang, and X. Ma, "Unfractionated heparin alleviates sepsis-induced acute lung injury by protecting tight junctions," The Journal of Surgical Research, vol. 238, pp. 175-185, 2019.

[33] A. Traweger, D. Fang, Y. C. Liu et al., "The Tight junctionspecific protein occludin is a functional target of the E3 ubiquitin-protein ligase Itch," The Journal of Biological Chemistry, vol. 277, no. 12, pp. 10201-10208, 2002.

[34] M. C. Nawijn, T. L. Hackett, D. S. Postma, A. J. M. van Oosterhout, and I. H. Heijink, "E-cadherin: gatekeeper of airway mucosa and allergic sensitization," Trends in Immunology, vol. 32, no. 6, pp. 248-255, 2011.

[35] G. W. Tu, M. J. Ju, Y. J. Zheng et al., "CXCL16/CXCR6 is involved in LPS-induced acute lung injury via P38 signalling," Journal of Cellular and Molecular Medicine, vol. 23, no. 8, pp. 5380-5389, 2019.

[36] Y. Fujita, G. Krause, M. Scheffner et al., "Hakai, a c-Cbl-like protein, ubiquitinates and induces endocytosis of the E-cadherin complex," Nature Cell Biology, vol. 4, no. 3, pp. 222-231, 2002.

[37] S. Dong, J. Liu, L. Li et al., "The HECT ubiquitin E3 ligase Smurf2 degrades $\mu$-opioid receptor 1 in the ubiquitinproteasome system in lung epithelial cells," American Journal of Physiology Cell Physiology, vol. 316, no. 5, pp. C632-C640, 2019.

[38] K. Bachmaier, S. Toya, X. Gao et al., "E3 ubiquitin ligase Cblb regulates the acute inflammatory response underlying lung injury," Nature Medicine, vol. 13, no. 8, pp. 920-926, 2007.

[39] J. Zhao, J. Wei, R. K. Mialki et al., "F-box protein FBXL19mediated ubiquitination and degradation of the receptor for IL-33 limits pulmonary inflammation," Nature Immunology, vol. 13, no. 7, pp. 651-658, 2012.

[40] B. B. Chen, T. A. Coon, J. R. Glasser et al., "A combinatorial F box protein directed pathway controls TRAF adaptor stability to regulate inflammation," Nature Immunology, vol. 14, no. 5, pp. 470-479, 2013.

[41] T. Suber, J. Wei, A. M. Jacko et al., " $\mathrm{SCF}^{\mathrm{FBXO17}}$ E3 ligase modulates inflammation by regulating proteasomal degradation of glycogen synthase kinase- $3 \beta$ in lung epithelia," The Journal of Biological Chemistry, vol. 292, no. 18, pp. 7452-7461, 2017.

[42] T. B. Lear, A. C. McKelvey, J. W. Evankovich et al., "KIAA0317 regulates pulmonary inflammation through SOCS2 degradation," JCI insight, vol. 4, no. 19, 2019.

[43] S. Hatakeyama, "TRIM family proteins: roles in autophagy, immunity, and carcinogenesis," Trends in Biochemical Sciences, vol. 42, no. 4, pp. 297-311, 2017.

[44] A. Reymond, G. Meroni, A. Fantozzi et al., "The tripartite motif family identifies cell compartments," The EMBO Journal, vol. 20, no. 9, pp. 2140-2151, 2001.

[45] Y. Li, X. Huang, F. Guo et al., "TRIM65 E3 ligase targets VCAM-1 degradation to limit LPS-induced lung inflammation," Journal of Molecular Cell Biology, vol. 12, no. 3, pp. 190-201, 2020. 
[46] B. A. Whitson, K. Mulier, H. Li et al., "MG53 as a novel therapeutic protein to treat acute lung injury," Military Medicine, vol. 186, Supplement_1, pp. 339-345, 2021.

[47] U. Bhaduri and G. Merla, "Rise of TRIM8: a molecule of duality," Molecular Therapy Nucleic Acids, vol. 22, pp. 434-444, 2020.

[48] D. Tomar, L. Sripada, P. Prajapati, R. Singh, A. K. Singh, and R. Singh, "Nucleo-cytoplasmic trafficking of TRIM8, a novel oncogene, is involved in positive regulation of TNF induced NF- $\kappa$ B pathway," PLoS One, vol. 7, no. 11, article e48662, 2012.

[49] Q. Li, J. Yan, A. P. Mao et al., “Tripartite motif 8 (TRIM8) modulates TNF $\alpha$ - and IL-1 -triggered NF- $\kappa \mathrm{B}$ activation by targeting TAK1 for K63-linked polyubiquitination," Proceedings of the National Academy of Sciences of the United States of America, vol. 108, no. 48, pp. 19341-19346, 2011.

[50] F. Marzano, L. Guerrini, G. Pesole, E. Sbisa, and A. Tullo, "Emerging roles of TRIM8 in health and disease," Cell, vol. 10, 2021.

[51] L. Li, Y. He, X. J. He, M. R. Bi, Y. H. Qi, and W. W. Zhu, "Down-regulation of long noncoding RNA LINC00472 alleviates sepsis-induced acute hepatic injury by regulating miR-373-3p/TRIM8 axis," Experimental and Molecular Pathology, vol. 117, p. 104562, 2020.

[52] L. Xiaoli, Z. Wujun, and L. Jing, "Blocking of tripartite motif 8 protects against lipopolysaccharide (LPS)-induced acute lung injury by regulating AMPK $\alpha$ activity," Biochemical and Biophysical Research Communications, vol. 508, no. 3, pp. 701708, 2019.

[53] T. Nagase, N. Seki, A. Tanaka, K. Ishikawa, and N. Nomura, "Prediction of the coding sequences of unidentified human genes. IV. The coding sequences of 40 new genes (KIAA0121KIAA0160) deduced by analysis of cDNA clones from human cell line KG-1," DNA Research, vol. 167-174, pp. 199-210, 1995.

[54] V. Tarantul, A. Nikolaev, H. Hannig et al., "Detection of abundantly transcribed genes and gene translocation in human immunodeficiency virus-associated non-Hodgkin's lymphoma," Neoplasia (New York, N.Y.), vol. 3, no. 2, pp. 132-142, 2001.

[55] V. Z. Tarantul, A. I. Nikolaev, A. Martynenko, H. Hannig, G. Hunsmann, and W. Bodemer, "Differential gene expression in B-cell non-Hodgkin's lymphoma of SIV-infected monkey," AIDS Research and Human Retroviruses, vol. 16, no. 2, pp. 173-179, 2000.

[56] R. Rajsbaum, A. García-Sastre, and G. A. Versteeg, “TRIMmunity: the roles of the TRIM E3-ubiquitin ligase family in innate antiviral immunity," Journal of Molecular Biology, vol. 426, no. 6, pp. 1265-1284, 2014.

[57] Y. Yu, L. Liang, Y. Jin, and Y. Yin, “The TRIM14 PRYSPRY domain mediates protein interaction via its basic interface," FEBS Letters, vol. 593, no. 10, pp. 1122-1129, 2019.

[58] M. Chen, Q. Meng, Y. Qin et al., “TRIM14 inhibits cGAS degradation mediated by selective autophagy receptor p62 to promote innate immune responses," Molecular Cell, vol. 64, no. 1, pp. 105-119, 2016.

[59] P. Tan, L. He, J. Cui et al., "Assembly of the WHIP-TRIM14PPP6C mitochondrial complex promotes RIG-I-mediated antiviral signaling," Molecular Cell, vol. 68, no. 2, article e295, pp. 293-307.e5, 2017.

[60] G. Xu, Y. Guo, D. Xu et al., “TRIM14 regulates cell proliferation and invasion in osteosarcoma via promotion of the AKT signaling pathway," Scientific Reports, vol. 7, no. 1, p. 42411, 2017.

[61] J. Hai, C. Q. Zhu, T. Wang, S. L. Organ, F. A. Shepherd, and M. S. Tsao, "TRIM14 is a putative tumor suppressor and regulator of innate immune response in non-small cell lung cancer," Scientific Reports, vol. 7, no. 1, p. 39692, 2017.

[62] Z. Tan, L. Song, W. Wu et al., "TRIM14 promotes chemoresistance in gliomas by activating $\mathrm{Wnt} / \beta$-catenin signaling via stabilizing Dvl2," Oncogene, vol. 37, no. 40, pp. 5403-5415, 2018.

[63] S. Feng, X. Cai, Y. Li, X. Jian, L. Zhang, and B. Li, “Tripartite motif-containing 14 (TRIM14) promotes epithelialmesenchymal transition via ZEB2 in glioblastoma cells," Journal of Experimental \& Clinical Cancer Research, vol. 38, no. 1, p. 57, 2019.

[64] J. Chen, L. Huang, J. Quan, and D. Xiang, “TRIM14 regulates melanoma malignancy via PTEN/PI3K/AKT and STAT3 pathways," Aging, vol. 13, no. 9, pp. 13225-13238, 2021.

[65] X. Huang, Y. Li, X. Li, D. Fan, H. B. Xin, and M. Fu, "TRIM14 promotes endothelial activation via activating NF- $\kappa \mathrm{B}$ signaling pathway," Journal of Molecular Cell Biology, vol. 12, no. 3, pp. 176-189, 2020.

[66] Z. Zhou, X. Jia, Q. Xue et al., "TRIM14 is a mitochondrial adaptor that facilitates retinoic acid-inducible gene-I-like receptor-mediated innate immune response," Proceedings of the National Academy of Sciences of the United States of America, vol. 111, no. 2, pp. E245-E254, 2014.

[67] M. Chen, Z. Zhao, Q. Meng et al., "TRIM14 promotes noncanonical NF- $\kappa$ B activation by modulating p100/p52 stability via selective autophagy," Advanced Science, vol. 7, no. 1, p. 1901261, 2020.

[68] K. Wada and T. Kamitani, "Autoantigen Ro52 is an E3 ubiquitin ligase," Biochemical and Biophysical Research Communications, vol. 339, no. 1, pp. 415-421, 2006.

[69] D. A. Rhodes, G. Ihrke, A. T. Reinicke et al., "The 52000 MW Ro/SS-A autoantigen in Sjogren's syndrome/systemic lupus erythematosus (Ro52) is an interferon-gamma inducible tripartite motif protein associated with membrane proximal structures," Immunology, vol. 106, no. 2, pp. 246-256, 2002.

[70] E. Ben-Chetrit, R. I. Fox, and E. M. Tan, "Dissociation of immune responses to the ss-a (ro) 52-kd and 60-kd polypeptides in systemic lupus erythematosus and sjögren's syndrome," Arthritis and Rheumatism, vol. 33, no. 3, pp. 349355, 1990.

[71] E. Ben-Chetrit, E. K. Chan, K. F. Sullivan, and E. M. Tan, “A $52-\mathrm{kD}$ protein is a novel component of the ss-a/ro antigenic particle," The Journal of Experimental Medicine, vol. 167, no. 5, pp. 1560-1571, 1988.

[72] S. Salomonsson, S. E. Sonesson, L. Ottosson et al., "Ro/SSA autoantibodies directly bind cardiomyocytes, disturb calcium homeostasis, and mediate congenital heart block," The Journal of Experimental Medicine, vol. 201, no. 1, pp. 11-17, 2005.

[73] A. H. Keeble, Z. Khan, A. Forster, and L. C. James, "TRIM21 is an IgG receptor that is structurally, thermodynamically, and kinetically conserved," Proceedings of the National Academy of Sciences of the United States of America, vol. 105, no. 16, pp. 6045-6050, 2008.

[74] D. L. Mallery, W. A. McEwan, S. R. Bidgood, G. J. Towers, C. M. Johnson, and L. C. James, "Antibodies mediate intracellular immunity through tripartite motif-containing 21 (TRIM21)," Proceedings of the National Academy of Sciences 
of the United States of America, vol. 107, no. 46, pp. 1998519990, 2010.

[75] M. Sjöstrand, A. Ambrosi, S. Brauner et al., "Expression of the immune regulator tripartite-motif 21 is controlled by IFN regulatory factors," Journal of Immunology, vol. 191, no. 7, pp. 3753-3763, 2013.

[76] H. Liu, M. Li, Y. Song, and W. Xu, "TRIM21 restricts Coxsackievirus B3 replication, cardiac and pancreatic injury via interacting with MAVS and positively regulating IRF3mediated type-I interferon production," Frontiers in Immunology, vol. 9, p. 2479, 2018.

[77] R. E. Watkinson, W. A. McEwan, J. C. H. Tam, M. Vaysburd, and L. C. James, "TRIM21 promotes cGAS and RIG-I sensing of viral genomes during infection by antibody-opsonized virus," PLoS Pathogens, vol. 11, no. 10, article e1005253, 2015.

[78] Z. Zhang, M. Bao, N. Lu, L. Weng, B. Yuan, and Y. J. Liu, "The E3 ubiquitin ligase TRIM21 negatively regulates the innate immune response to intracellular double-stranded DNA," Nature Immunology, vol. 14, no. 2, pp. 172-178, 2013.

[79] S. Foss, M. Bottermann, A. Jonsson, I. Sandlie, L. C. James, and J. T. Andersen, "TRIM21-from intracellular immunity to therapy," Frontiers in Immunology, vol. 10, p. 2049, 2019.

[80] R. Yoshimi, T. H. Chang, H. Wang, T. Atsumi, H. C. Morse III, and K. Ozato, "Gene disruption study reveals a nonredundant role for TRIM21/Ro52 in NF-kappaB-dependent cytokine expression in fibroblasts," Journal of Immunology, vol. 182, no. 12, pp. 7527-7538, 2009.

[81] A. Espinosa, V. Dardalhon, S. Brauner et al., "Loss of the lupus autoantigen Ro52/Trim21 induces tissue inflammation and systemic autoimmunity by disregulating the IL-23-Th17 pathway," The Journal of Experimental Medicine, vol. 206, no. 8, pp. 1661-1671, 2009.

[82] L. Li, J. Wei, R. K. Mallampalli, Y. Zhao, and J. Zhao, "TRIM21 mitigates human lung microvascular endothelial cells' inflammatory responses to LPS," American Journal of Respiratory Cell and Molecular Biology, vol. 61, no. 6, pp. 776-785, 2019.

[83] V. Natarajan, "Mind the gap between the endothelium and E3 ubiquitin ligase: TRIM21 is a viable therapeutic target in sepsis-induced endothelial dysfunction," American Journal of Respiratory Cell and Molecular Biology, vol. 61, no. 6, pp. 676-677, 2019.

[84] H. Schmidt, P. Freudenberger, S. Seiler, and R. Schmidt, "Genetics of subcortical vascular dementia," Experimental Gerontology, vol. 47, no. 11, pp. 873-877, 2012.

[85] P. Freudenberger, R. Schmidt, and H. Schmidt, "Genetics of age-related white matter lesions from linkage to genome wide association studies," Journal of the Neurological Sciences, vol. 322, no. 1-2, pp. 82-86, 2012.

[86] S. Li, L. Wang, B. Fu, and M. E. Dorf, "Trim65: a cofactor for regulation of the microRNA pathway," RNA Biology, vol. 11, no. 9, pp. 1113-1121, 2014.

[87] S. Li, L. Wang, B. Fu, M. A. Berman, A. Diallo, and M. E. Dorf, "TRIM65 regulates microRNA activity by ubiquitination of TNRC6," Proceedings of the National Academy of Sciences of the United States of America, vol. 111, no. 19, pp. 6970-6975, 2014.

[88] X. Lang, T. Tang, T. Jin, C. Ding, R. Zhou, and W. Jiang, "TRIM65-catalized ubiquitination is essential for MDA5mediated antiviral innate immunity," The Journal of Experimental Medicine, vol. 214, no. 2, pp. 459-473, 2017.
[89] J. Meng, Z. Yao, Y. He et al., “ARRDC4 regulates enterovirus 71 -induced innate immune response by promoting K63 polyubiquitination of MDA5 through TRIM65," Cell Death \& Disease, vol. 8, no. 6, article e2866, 2017.

[90] Y. Li, C. Ma, T. Zhou, Y. Liu, L. Sun, and Z. Yu, “TRIM65 negatively regulates p53 through ubiquitination," Biochemical and Biophysical Research Communications, vol. 473, no. 1, pp. 278-282, 2016.

[91] W. S. Wei, X. Chen, L. Y. Guo et al., “TRIM65 supports bladder urothelial carcinoma cell aggressiveness by promoting ANXA2 ubiquitination and degradation," Cancer Letters, vol. 435, pp. 10-22, 2018.

[92] Y. F. Yang, M. F. Zhang, Q. H. Tian, and C. Z. Zhang, "TRIM65 triggers $\beta$-catenin signaling via ubiquitylation of Axin1 to promote hepatocellular carcinoma," Journal of Cell Science, vol. 130, no. 18, pp. 3108-3115, 2017.

[93] D. Chen, Y. Li, X. Zhang et al., "Ubiquitin ligase TRIM65 promotes colorectal cancer metastasis by targeting ARHGAP35 for protein degradation," Oncogene, vol. 38, no. 37, pp. 6429-6444, 2019.

[94] B. Liu, Y. Tang, P. Yang, C. Wu, and Y. Huang, "TRIM65 in white matter lesions, innate immunity and tumor," Current Molecular Pharmacology, vol. 14, 2021.

[95] J. Wang, X. Liang, T. Yu et al., "TRIM65 is a potential oncogenic protein via ERK1/2 on Jurkat and Raji cells: a therapeutic target in human lymphoma malignancies," Cell Biology International, vol. 42, no. 11, pp. 1503-1510, 2018.

[96] Y. T. Wu, S. Y. Ma, W. Q. Sun et al., "TRIM65 promotes invasion of endometrial stromal cells by activating ERK1/2/Cmyc signaling via ubiquitination of DUSP6," The Journal of Clinical Endocrinology and Metabolism, vol. 106, no. 2, pp. 526-538, 2021.

[97] Y. Jia, K. Chen, P. Lin et al., "Treatment of acute lung injury by targeting MG53-mediated cell membrane repair," Nature Communications, vol. 5, no. 1, 2014.

[98] M. Sermersheim, A. D. Kenney, P. H. Lin et al., "MG53 suppresses interferon- $\beta$ and inflammation via regulation of ryanodine receptor-mediated intracellular calcium signaling," Nature Communications, vol. 11, no. 1, p. 3624, 2020.

[99] P. Duann, H. Li, P. Lin et al., "MG53-mediated cell membrane repair protects against acute kidney injury," Science Translational Medicine, vol. 7, no. 279, p. 279ra236, 2015.

[100] C. S. Lee, J. S. Yi, S. Y. Jung et al., “TRIM72 negatively regulates myogenesis via targeting insulin receptor substrate-1," Cell Death and Differentiation, vol. 17, no. 8, pp. 12541265, 2010.

[101] C. Cai, H. Masumiya, N. Weisleder et al., "MG53 nucleates assembly of cell membrane repair machinery," Nature Cell Biology, vol. 11, no. 1, pp. 56-64, 2009.

[102] M. Hwang, J. K. Ko, N. Weisleder, H. Takeshima, and J. Ma, "Redox-dependent oligomerization through a leucine zipper motif is essential for MG53-mediated cell membrane repair," American Journal of Physiology Cell Physiology, vol. 301, no. 1, pp. C106-C114, 2011.

[103] M. K. Ahn, K. J. Lee, C. Cai et al., "Mitsugumin 53 regulates extracellular $\mathrm{Ca}^{2+}$ entry and intracellular $\mathrm{Ca}^{2+}$ release via Orail and RyR1 in skeletal muscle," Scientific Reports, vol. 6, no. 1, p. 36909, 2016.

[104] T. Tan, Y. G. Ko, and J. Ma, "Dual function of MG53 in membrane repair and insulin signaling," BMB Reports, vol. 49, no. 8, pp. 414-423, 2016. 
[105] Z. Li, L. Wang, H. Yue et al., "MG53, a tissue repair protein with broad applications in regenerative medicine," Cell, vol. 10, no. 1, p. 122, 2021.

[106] A. D. Kenney, Z. Li, Z. Bian et al., "Recombinant MG53 protein protects mice from lethal influenza virus infection," American Journal of Respiratory and Critical Care Medicine, vol. 203, no. 2, pp. 254-257, 2021. 